\title{
Item Group Definition
}

National Cancer Institute

\section{Source}

National Cancer Institute. Item Group Definition. NCI Thesaurus. Code C142593.

The formal specification of the properties of a group of related items. 